\title{
The polymorphism of XRCC1 Arg399Gln (rs25487) and male infertility risk: a meta-analysis of 1,407 cases and 974 control studies
}

\author{
Ibrahimi $\mathrm{R}^{1}$, Musavi $\mathrm{M}^{2}$, Shojaee $\mathrm{M}^{3}$, Moossavi $\mathrm{M}^{4,5}$, Moossavi $\mathrm{SZ}^{6}$, Alaei $\mathrm{M}^{7}$, Poodineh $\mathrm{J}^{8}$, \\ Ibrahimi $\mathrm{M}^{7}$, Farahani $\mathrm{Y}^{7}$, Khorasani $\mathrm{M}^{7}$
}

Department of Clinical Biochemistry, Faculty of Medical Sciences, Tarbiat Modares University, Tehran, Iran. milad.kh@mofares.ac.ir

\begin{abstract}
X-ray repair cross-complementing group 1 (XRCC1) is a scaffold protein and a key element in DNA base excision repair process. Although, the role of XRCC1 polymorphisms in male infertility has been studied broadly, it is still a matter of debate. Hence, in order to shed light on the problem, we performed a meta-analysis to evaluate the overall effect of XRCC1 polymorphisms in male infertility risk.

Databases, Web of Science, PubMed, Scopus, and Google Scholar were searched until September 15, 2018. Afterwards, the genotypes' distribution, genotyping methods, and ethnicity groups were extracted, and overall analyses were conducted.

A total number of five researches on 1,407 subjects and 974 controls were found to meet our criteria in this meta-analysis. The XRCC1 Arg399GIn (rs25487) polymorphism was analyzed. This is the first meta-analysis to investigate the association of XRCC1 polymorphisms (codon 399) and male infertility risk. Our results indicated that the XRCC1 Arg399GIn polymorphism was not associated with male infertility risk in the total studied populations (Tab. 2, Fig. 3, Ref. 26). Text in PDF www.elis.sk.

KEY WORDS: meta-analysis; male infertility; polymorphism; XRCC1 Arg399GIn.
\end{abstract}

\section{Introduction}

Infertility is defined as an inability to achieve clinical pregnancy after 12 or more months of consistent unprotected intercourse $(1,2)$. Infertility is categorized into primary and secondary types. Primary infertility is the occasion when no previous birth has occurred, or the woman is unable to become pregnant. Most infertilities are of primary type (67-71\%). Secondary infertility is involved when a couple have been fertile but they are no more, which includes about $29-33 \%$ of cases (3). It is reported that $7 \%$ of men at reproductive age are suffering from male inferti-

${ }^{1}$ Research Committee Faculty of Medicine, Mashhad University of Medical Sciences Mashhad, ${ }^{2}$ Department of Biostatistics \& Epidemiology, Hamadan University of Medical Sciences, Hamadan, Iran, ${ }^{3}$ Department of Biology, Payame Noor University of Mashhad, IR Iran, ${ }^{4}$ Student Research Committee, Birjand University of Medical Sciences, Birjand, Iran, ${ }^{5}$ Department of Molecular Medicine, Birjand University of Medical Sciences, Birjand, Iran, ${ }^{6}$ Shiraz University of Medical Sciences, Shiraz, IR Iran, ${ }^{7}$ Department of Clinical Biochemistry, Faculty of Medical Sciences, Tarbiat Modares University, Tehran, IR Iran, and ${ }^{8}$ Department of Clinical Biochemistry, School of Medicine, Zabol University of Medical Sciences, Zabol, IR Iran Reza Ibrahimi, Mahsa Musavi, Maryam Shojaee and Maryam Moossavi, equally contributed as first author.

Address for correspondence: M. Khorasani, Department of Clinical Biochemistry, Faculty of Medical Sciences, Tarbiat Modares University, Tehran, Iran.

Phone: +98.9155000128 lity. Moreover, in about $50 \%$ of infertile men, the basic cause of their infertility is not defined (4). Nevertheless, in many of the cases, the male infertility is due to poor quality of sperm which is a complicated medical condition rooted in a variety of etiologies. Besides environmental factors, genetics is the main factor of male infertility. The genetic background is considered to be the corner stone in $15-30 \%$ of male infertility cases (4). The impact of genetic factors on infertility has been reported in a variety of studies on specific genes in both, human and experimental models (5). As a result of high levels of reactive oxygen species (ROS) production in the course of spermatogenesis (6), medical complications such as varicocele (7), and effects of environmental elements and drugs, the genomic integrity of sperm is highly prone to be damaged (8). As a result, the DNA damage in somatic and germ cells may lead to azoospermia (9). It is also verified that the DNA damage is more common in patients with complete spermatogenesis failure, compared to patients with incomplete spermatogenesis failure (10). Furthermore, higher levels of sperm DNA damage and chromosomal fragility have been reported in infertile men in comparison with the healthy control group (11). Hence, sperm DNA integrity maintenance and DNA repair seem to be of paramount importance for male fertility. Two major types of DNA damage includes single-strand breaks (SSB) and double-strand breaks which are caused by a variety of factors such as reactive oxygen species, radiation exposure, chemical materials and alkylation (12). Oxidative stress can alter sperm DNA through different mechanisms, one of which 
is the oxidation of guanine bases that results in the production of 8 -hydroxy-2'-deoxyguanosine (8-OHdG). The $8-\mathrm{OHdG}$ is a mutagenic metabolite causing $\mathrm{G}: \mathrm{C} \rightarrow \mathrm{T}:$ A transversion after replication (13). Localized DNA damage correction relies on the base excision repair (BER) pathway. BER is an important system that corrects damage caused by methylation or oxidation, or removes fragmented lesions of DNA and non-bulky adducts that can cause genetic instability or blockage of DNA replication (14). DNA repair enzymes check the chromosomes constantly for faulty nucleotides caused by methylation, or oxidative damage, and tend to correct them. (15) X-ray repair cross-complementing group 1 (XRCC1) is a scaffolding protein involved in several DNA repair systems and plays a central role in BER. The XRCC1 gene is located on chromosome 19q13.2 and holds 17 exons (16). Although numerous epidemiological studies have been conducted to investigate the association between XRCC1 SNPs and risk of male infertility, the relation remains unclear. The genetic heterogeneity of studied participants, inadequate statistical power in individual studies, and so forth, can be the source of such uncertainty. Ergo, this metaanalysis is performed to thoroughly investigate the evidence for the association between XRCC1 Arg399Gln (rs25487) polymorphism and risk of male infertility.

\section{Methods}

\section{Literature search}

A search through the Web of Science, PubMed, Scopus, and Google Scholar databases was carried out in order to analyze all publications released up to September 15, 2018 and contributing to the knowledge on the association between XRCC1 Arg399Gln

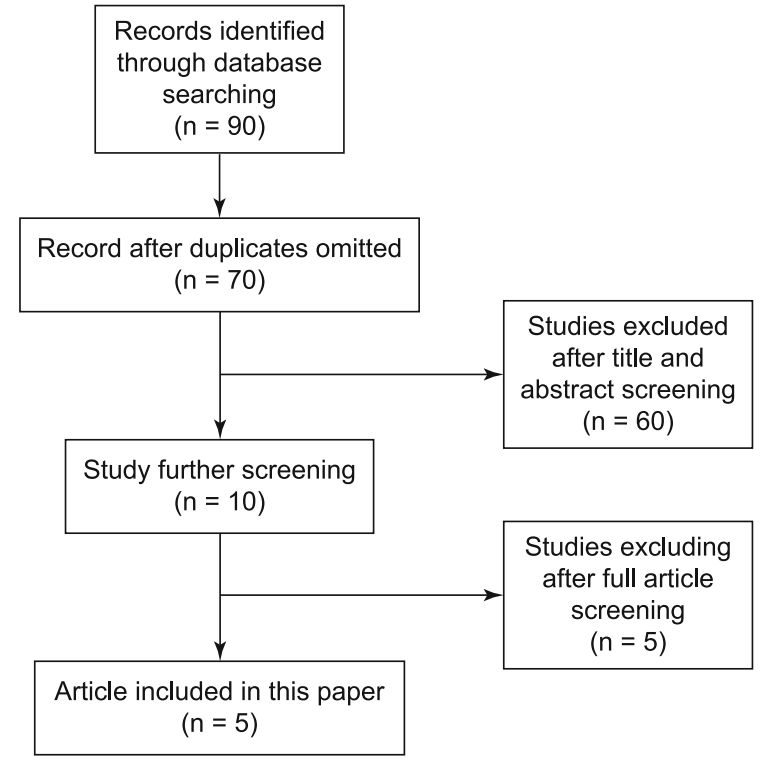

Fig. 1. Flow chart showing the detailed steps for literature selection.

(rs25487) polymorphism and male infertility risk. The search strategy was "male infertility OR infertility" AND "XRCC1 OR XRCC1 Arg399Gln” AND “polymorphism OR mutation OR variant OR rs25487". Articles were included in the meta-analysis if they were in accordance with inclusion criteria as follows: 1) original case-control studies evaluating the association between XRCC1 polymorphism and male infertility; 2) articles that con-

Tab. 1. Main characteristics of studies included in the meta-analysis.

\begin{tabular}{|c|c|c|c|c|c|c|c|c|}
\hline Authors & Year & Country & Sample & Method & CaseAA/AG/GG & ControlAA/AG/GG & HW-p & HW-adj.p \\
\hline Garcia-Rodriguez et al (19) & 2018 & Spain & Seminal samples & PCR-RFLP & $40 / 129 / 144$ & $13 / 45 / 22$ & 0.2124 & 0.354 \\
\hline Ghasemi et al (18) & 2017 & Iran & Blood & PCR-RFLP & $7 / 106 / 78$ & $8 / 91 / 92$ & 0.0122 & 0.061 \\
\hline Zheng et al (17) & 2012 & China & Blood & PCR-RFLP & $12 / 67 / 33$ & $15 / 69 / 72$ & 0.7939 & 0.7939 \\
\hline Gu et al (20) & 2007 & China & Blood & PCR-RFLP & $5 / 64 / 102$ & $21 / 91 / 135$ & 0.3167 & 0.3959 \\
\hline Ji et al (21) & 2010 & China & Blood & PCR-RFLP & $54 / 339 / 327$ & $23 / 97 / 153$ & 0.1809 & 0.354 \\
\hline
\end{tabular}

Tab. 2. Results of the meta-analysis for the association between the XRCC1 and male infertility.

\begin{tabular}{|c|c|c|c|c|c|c|c|}
\hline \multirow{2}{*}{ Allelic and genotypic } & \multirow{2}{*}{ No. of studies } & \multicolumn{2}{|l|}{ Odds ratio } & \multirow{2}{*}{ Model } & \multicolumn{2}{|c|}{ Heterogeneity } & \multirow{2}{*}{$\mathrm{p}$ (Egger's test) } \\
\hline & & OR $[95 \% \mathrm{CI}]$ & $\mathrm{p}$ & & $\mathrm{I}^{2}$ & $\mathrm{p}$ & \\
\hline A vs. G & 6 & $1.0003[0.87 ; 1.144]$ & 0.996 & Fixed effect & 0.7473 & 0.0033 & 0.6835 \\
\hline A vs. $\mathrm{G}$ & 6 & $0.9819[0.7456 ; 1.293]$ & 0.896 & Random effect & & & \\
\hline AA vs. AG+GG & 6 & $0.8499[0.6144 ; 1.1756]$ & 0.3257 & Fixed effect & 0.1636 & 0.3104 & 0.3152 \\
\hline AA vs. AG+GG & 6 & $0.8332[0.578 ; 1.2002]$ & 0.3270 & Random effect & & & \\
\hline $\mathrm{AA}+\mathrm{AG}$ vs. GG & 6 & $1.066[0.8938 ; 1.2733]$ & 0.4736 & Fixed effect & 0.7959 & 0.0006 & 0.766 \\
\hline $\mathrm{AA}+\mathrm{AG}$ vs. GG & 6 & $1.0373[1.6895 ; 1.5606]$ & 0.8603 & Random effect & & & \\
\hline $\mathrm{AG}$ vs. $\mathrm{AA}+\mathrm{GG}$ & 6 & $1.1231[0.9409 ; 1.3405]$ & 0.1986 & Fixed effect & 0.7033 & 0.0091 & 0.8448 \\
\hline $\mathrm{AG}$ vs. $\mathrm{AA}+\mathrm{GG}$ & 6 & $1.1105[0.7941 ; 1.5528]$ & 0.5402 & Random effect & & & \\
\hline AA vs. GG & 6 & $0.8596[0.6105 ; 1.2104]$ & 0.3862 & Fixed effect & 0.5877 & 0.0458 & 0.5787 \\
\hline AA vs. GG & 6 & $0.8069[0.458 ; 1.4216]$ & 0.4577 & Random effect & & & \\
\hline AA vs. AG & 6 & $0.8324[0.5928 ; 1.1688]$ & 0.2895 & Fixed effect & 0 & 0.4456 & 0.1961 \\
\hline AA vs. AG & 6 & $0.8324[0.5928 ; 1.1688]$ & 0.2895 & Random effect & & & \\
\hline AG vs. GG & 6 & $1.1088[0.9219 ; 1.3336]$ & 0.2726 & Fixed effect & 0.7774 & 0.0012 & 0.7501 \\
\hline AG vs. GG & 6 & $1.0782[0.7179 ; 1.6193]$ & 0.716 & Random effect & & & \\
\hline
\end{tabular}


A vs. G

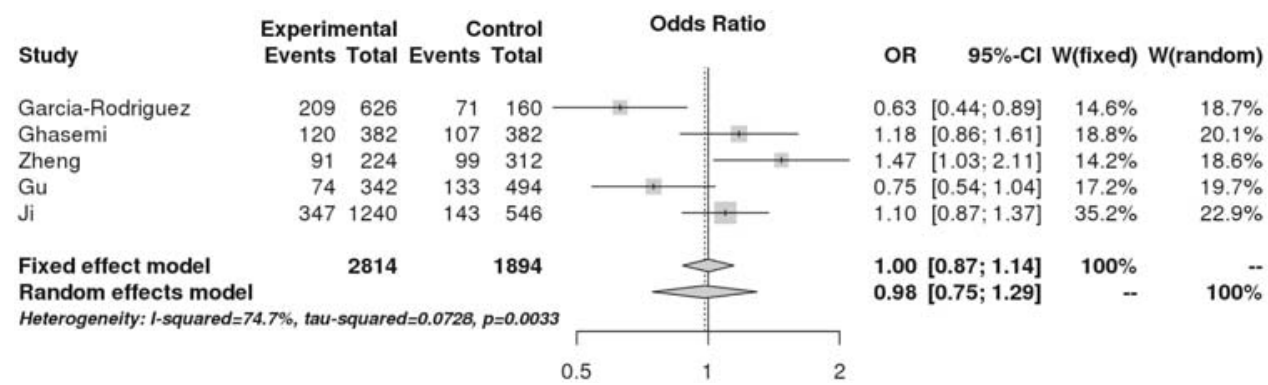

$A A$ vs. $A G+G G$

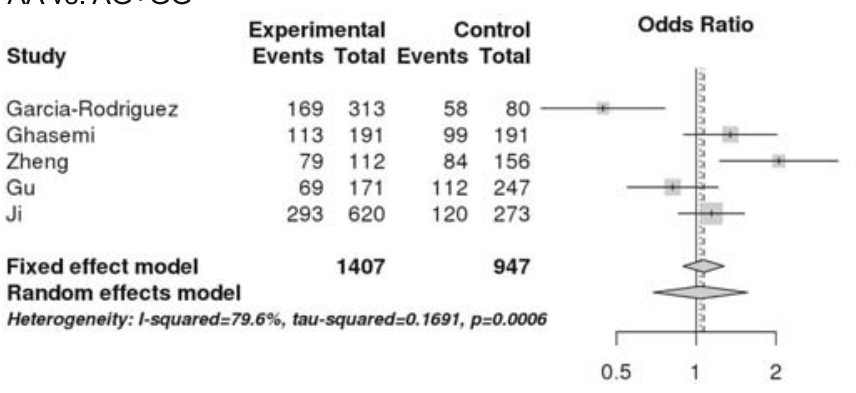

OR $\quad 95 \%-\mathrm{Cl}$ W(fixed) W(random)

$\begin{array}{rrr}0.45[0.26 ; 0.76] & 10.8 \% & 17.7 \% \\ 1.35[0.90 ; 2.02] & 19.1 \% & 20.5 \% \\ 2.05[1.23 ; 3.43] & 11.9 \% & 18.3 \% \\ 0.82[0.55 ; 1.21] & 20.1 \% & 20.7 \% \\ 1.14[0.86 ; 1.52] & 38.2 \% & 22.8 \% \\ & & - \\ \mathbf{1 . 0 7}[\mathbf{0 . 8 9} ; \mathbf{1 . 2 7}] & \mathbf{1 0 0} \% & \mathbf{1 0 0} \% \\ \mathbf{1 . 0 4}[\mathbf{0 . 6 9} \mathbf{1 . 5 6}] & -- & \mathbf{1 0 0}\end{array}$

$A A+A G$ vs. $G G$

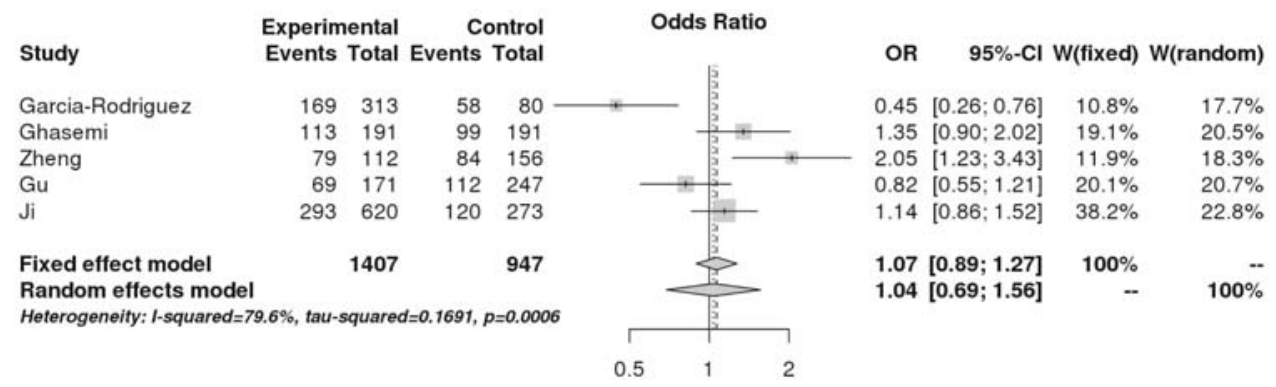

$A G$ vs. $A A+G G$

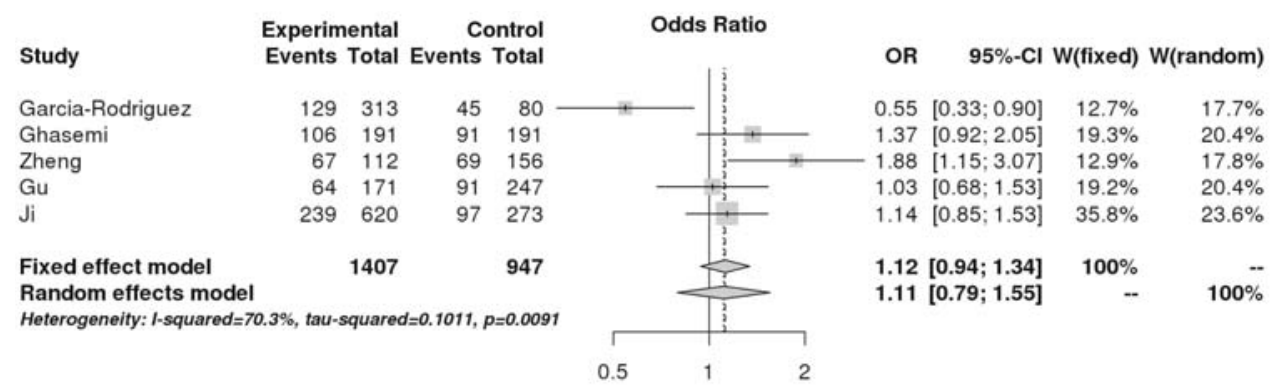

AA vs. GG

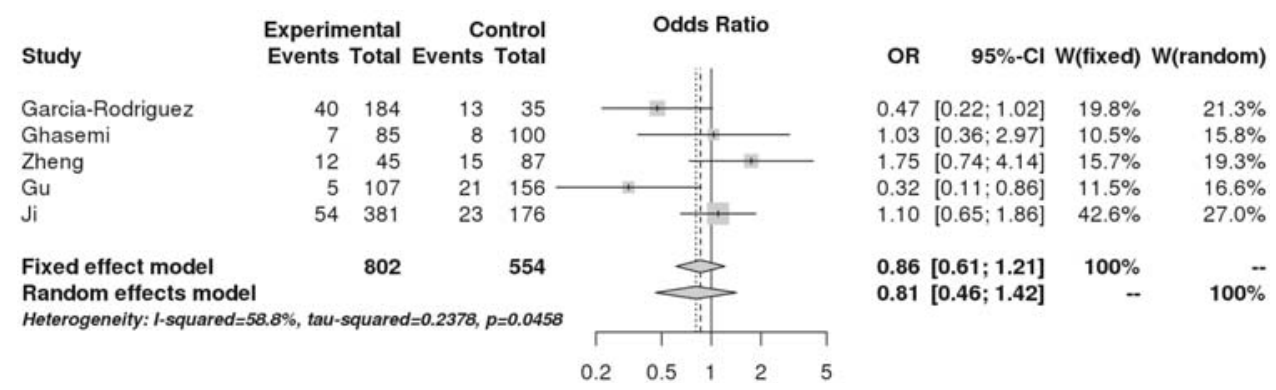




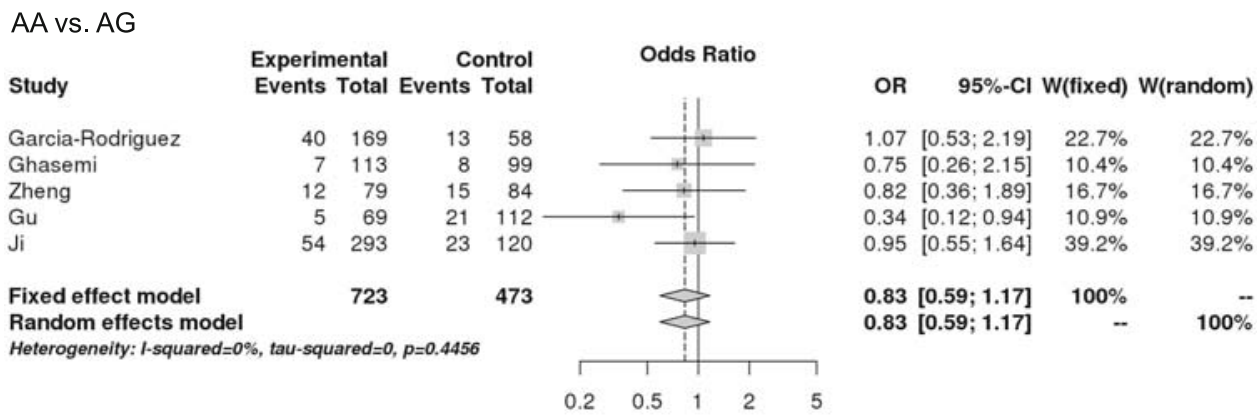

AG vs. GG

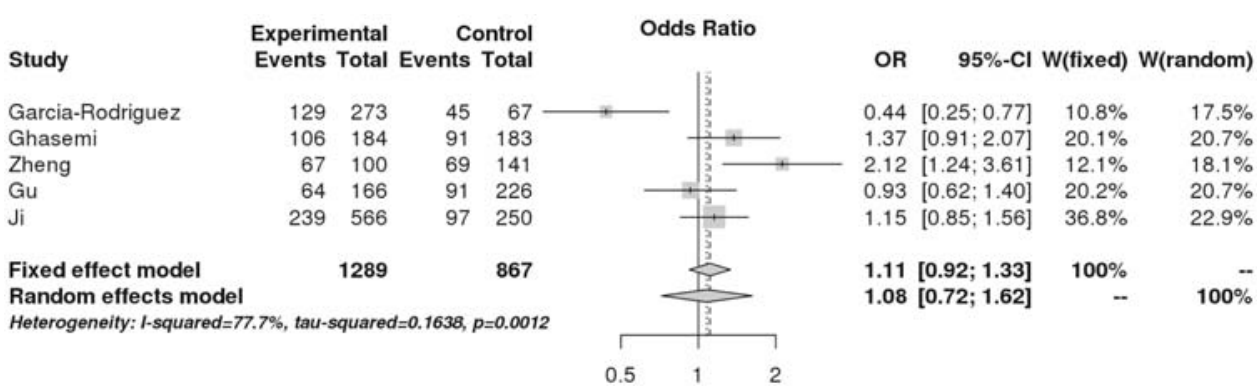

Fig. 2. Forest plots for the association between XRCC1 Arg399GIn SNP and male infertility risk.

tribute with necessary data of the genotype frequencies of the XRCC1 Arg399Gln rs25487 variant in both cases and controls. The exclusion criteria were as follows: 1) conference abstract, case reports, reviews, and duplication data; 2) insufficient genotype information. Also, the search investigated only the studies carried out on human subjects.

\section{Data extraction}

Upon the through appraisal of all articles meeting the inclusion and exclusion criteria, the relevant information was extracted. Afterwards, each study exploited variables as follows: first author's name, year of publication, country, genotyping method and sample type, sample size of the studied case and control groups, and results of the Hardy-Weinberg equilibrium test.

\section{Statistical analysis}

The significance of the relationships between XRCC1 Arg399Gln (rs25487) polymorphism and risk of male infertility risks were evaluated by means of OR and corresponding $95 \%$ CI. The net ORs were used for allele comparison model, dominant model, recessive model and codominant model. Chi-square-based $\mathrm{Q}$ test was then performed in order to test the heterogeneity assumption. The value of $\mathrm{p}<0.10$ was considered to represent significant heterogeneity, and $\mathrm{I}^{2}$ values of $25 \%, 50 \%$ and $75 \%$ contributed to low, medium and high levels of heterogeneity, respectively. A fixed-effect model was used to assess the net OR, when the $p$ value for heterogeneity was $>0.10$ and $\mathrm{I}^{2}<50 \%$. Conversely, if $\mathrm{p} \leq 0.10$ or $\mathrm{I}^{2} \geq 50 \%$, we performed the random-effect model. The significance of the net OR was set on by the Z-test, and $p<0.05$ was perceived as statistically significant. The statistical analysis was carried out by means of Reviewer Manager 5.3 and Stata 12.0.
The potential publication bias was estimated using Egger's test and funnel plots. For the purpose of appraising the stability of the result, the sensitivity analysis was performed. The net ORs were calculated by excluding individual study, one at a time, to assess the significance of a single study.

\section{Results}

Upon primary search through scientific databases of PubMed, Scopus, Google Scholar and Web of Science, 90 results were retrieved. Of those chosen, 10 studies were excluded based on the screening criteria. Finally, after careful consideration, 5 casecontrol studies with a total of 1,407 cases and 974 controls were chosen and further investigated in this meta-analysis (17-21) (Fig. 1). These studies were published between years 2007 to 2018 . All included studies were assessed performing the Hardy-Weinberg test (HWE) to evaluate all the included data, and the results confirmed that the XRCC1 Arg399Gln gene genotype frequencies of all five studies were in HWE in the controls. The elaborated characteristics of all included data are shown in Table 1.

\section{XRCC1 polymorphism and male infertility risk}

A total of 2,381 individuals in five studies were included, in which the influence of XRCC1 Arg399Gln (rs25487) polymorphism on the risk of male infertility were evaluated. The results of meta-analyses on the associations between XRCC1 Arg399Gln polymorphism and male infertility risk is summarized in Table 2.

As shown in Table 2, there is no significant relation between the XRCC1 genotype and male infertility. The forest plots are shown in Figure 2. 

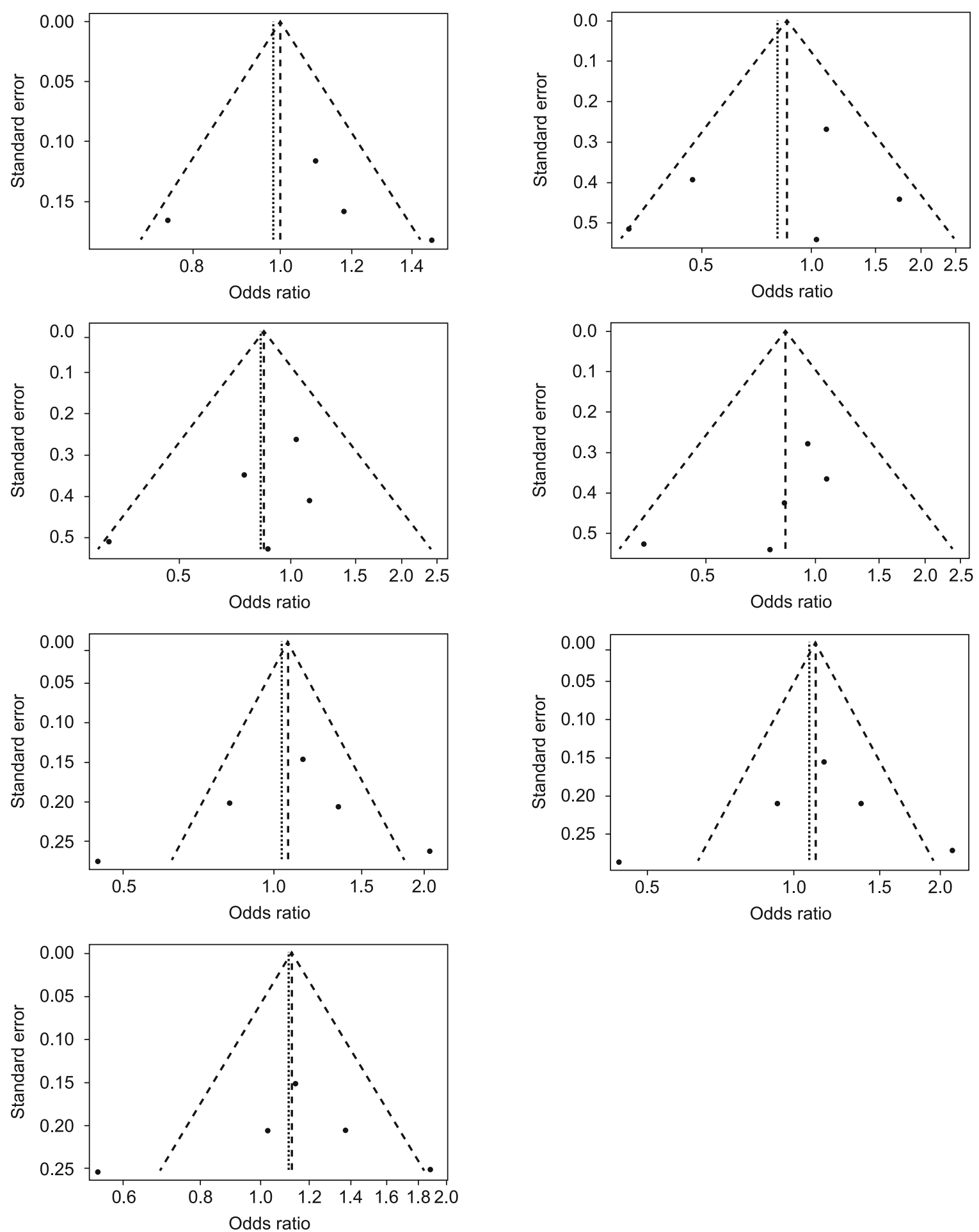

Fig. 3. Funnel plots of the association between cancer risk and rs25487 polymorphism in the overall study population.

Heterogeneity analysis

Overall comparisons approved the heterogeneity among studies (Tab. 2). To detect the risk of publication bias, a funnel plot was deployed as a visual aid (Fig. 3). Regarding the rs25487 variant,
Egger's linear regression analysis showed no publication bias for this meta-analysis of the codominant, dominant, recessive, overdominant and allele model (all $\mathrm{p}$ values for bias $<0.05$ ). 


\section{Discussion}

Since 2007, several case-control studies have found a specific association between XRCC1 polymorphisms and increased risk of male infertility (20). In this meta-analysis, 5 relevant studies were screened. While two of them demonstrate a significant association between XRCC1 polymorphisms and male infertility $(17,19)$, the other selected studies do not show any notable association $(18,20,21)$. Hence, this meta-analysis was performed to evaluate the association of XRCC1 and male infertility in a comprehensive manner. We found out that Arg399Gln polymorphisms of the XRCC1 gene was not associated with male infertility risk in studied populations. No specific etiology is found in more than half of male infertility cases. In addition, a large proportion of male infertility is believed to be accompanied with idiopathic azoospermia. Idiopathic azoospermia is considered to result from changes in the DNA including microdeletions in Y chromosome, specific gene mutations and other chromosomal abnormalities (22). Nevertheless, there is no doubt that genetic polymorphisms might be also contributing to susceptibility to some forms of male infertility $(23,24)$. During spermatogenesis high amounts of reactive oxygen species are produced in the testes, which can cause several forms of DNA lesions (6) Moreover, agricultural and industrial chemicals as well as some drugs can damage the DNA in spermatogenic cells. Hence, interruptions in DNA repair mechanisms may be associated with the decrease in sperm count or production of abnormal sperms (25). Additionally, it was shown that polymorphism of DNA repair gene BRCA2 is in fact associated with idiopathic azoospermia (26). XRCC1, a necessary gene in the BER pathway, has a crucial part in single-strand breaks repair in meiotic recombination during spermatogenesis (15). Clearly, the publication bias is an important factor concerning the reliability of results, while the latter together with the study quality are crucial in conducting a meta-analytic study. We used Begg's funnel plots and Egger's test to analyze the publication bias in this study. Doing so, no significant publication bias was detected towards the reliability of our results. Same results were obtained using sensitivity analysis. Furthermore, we employed strict inclusion and exclusion criteria to diminish the selection bias. Although, this is an up-to-date meta-analysis, it suffers some limitations. Firstly, only selected databases were used to obtain data. Ergo, the publication might have been restricted. It is possible that some unpublished investigations with unidentified findings were missed. Secondly, as only Chinese, Iranian and Spanish populations were studied in our selected literature, the results might not be applicable to other ethnic populations. Hence, more studies especially those of different ethnicities might grant more solid evidence towards our questions. And thirdly, our analysis was based only on studies published in English.

\section{Conclusions}

At the time of conducting the present study, this was the first meta-analysis to investigate the association of XRCC1 polymorphisms (codon 399) and male infertility risk. Our results indicated that the XRCC1 Arg399Gln polymorphism was not associated with male infertility risk. More case-control investigations are needed to validate our finding.

\section{References}

1. Abhari A, Zarghami N, Shahnazi V, Barzegar A, Farzadi L, Karami H, Vahed SZ, Nouri M. Significance of microRNA targeted estrogen receptor in male fertility. Iranian J Basic Med Sci 2014; 17: 81.

2. Gholinezhad M, Yousefnia-pasha Y, Hosseinzadeh Colagar A, Mohammadoo-Khorasani M, Bidmeshkipour A. Comparison of large-scale deletions of the sperm mitochondrial DNA in normozoospermic and asthenoteratozoospermic men. J Cell Biochem 2018.

3. Jamalvandi M, Motovali-bashi M, Amirmahani F, Darvishi P, Goharrizi KJ. Association of T/A polymorphism in miR-1302 binding site in CGA gene with male infertility in Isfahan population. Mol Biol Rep 2018: $1-5$.

4. Carrell DT, Emery BR, Hammoud S. The aetiology of sperm protamine abnormalities and their potential impact on the sperm epigenome. Int J Androl 2008; 31: 537-545.

5. Ma X, Kuete M, Gu X, Zhou H, Xiong C, Li H. Recurrent deletions of the X chromosome linked CNV64, CNV67, and CNV69 shows geographic differences across China and no association with idiopathic infertility in men. PLoS One 2017; 12: e0185084.

6. Fisher HM, Aitken RJ. Comparative analysis of the ability of precursor germ cells and epididymal spermatozoa to generate reactive oxygen metabolites. J Exp Zool 1997; 277: 390-400.

7. Koksal I, Usta M, Orhan I, Abbasoglu S, Kadioglu A. Potential role of reactive oxygen species on testicular pathology associated with infertility. Asian J Androl 2003; 5: 95-100.

8. Morris ID. Sperm DNA damage and cancer treatment 1. Int J Androl 2002; 25: 255-261.

9. Said TM, Paasch U, Glander HJ, Agarwal A. Role of caspases in male infertility. Hum Reprod Update 2004; 10: 39-51.

10. Gu A, Ji G, Liang J, Xia Y, Lu N, Wu B, Wang W, Song L, Wang $\mathrm{S}$, Wang $\mathrm{X}$. DNA repair gene XRCC1 and XPD polymorphisms and the risk of idiopathic azoospermia in a Chinese population. Int J Mol Med 2007; 20: 743-747.

11. Papachristou F, Lialiaris T, Touloupidis S, Kalaitzis C, Simopoulos C, Sofikitis N. Evidence of increased chromosomal instability in infertile males after exposure to mitomycin $\mathrm{C}$ and caffeine. Asian J Androl 2006; 8: 199-204.

12. Lieber MR. The mechanism of double-strand DNA break repair by the nonhomologous DNA end-joining pathway. Ann Rev Biochem 2010; 79: 181-211.

13. Wood ML, Esteve A, Morningstar ML, Kuziemko GM, Essigmann JM. Genetic effects of oxidative DNA damage: comparative mutagenesis of 7, 8-dihydro-8-oxoguanine and 7, 8-dihydro-8-oxoadenine in Escherichia coli. Nucleic Acids Res 1992; 20: 6023-6032.

14. Wood RD, Mitchell M, Sgouros J, Lindahl T. Human DNA repair genes. Science 2001; 291: 1284-1289.

15. Salimi S, Mohammadoo-Khorasani M, Tabatabai E, Sandoughi M, Zakeri Z, Naghavi A. XRCC1 Arg399Gln and Arg194Trp polymorphisms and risk of systemic lupus erythematosus in an Iranian population: a pilot study. BioMed Res Internat 2014. 
16. Langsenlehner T, Renner W, Gerger A, Hofmann G, Thurner EM, Kapp KS, Langsenlehner U. Association between single nucleotide polymorphisms in the gene for XRCC1 and radiation-induced late toxicity in prostate cancer patients. Radiother Oncol 2011; 98: 387-393.

17. Zheng LR, Wang XF, Zhou DX, Zhang J, Huo YW, Tian H. Association between XRCC1 single-nucleotide polymorphisms and infertility with idiopathic azoospermia in northern Chinese Han males. Reprod Biomed Online 2012; 25: 402-407.

18. Ghasemi H, Khodadadi I, Fattahi A, Moghimbeigi A, Tavilani H. Polymorphisms of DNA repair genes XRCC1 and LIG4 and idiopathic male infertility. Syst Biol Reprod Med 2017; 63: 382-390.

19. Garcia-Rodriguez A, de la Casa M, Serrano M, Gosálvez J, Barcelona RR. Impact of polymorphism in DNA repair genes OGG1 and XRCC1 on seminal parameters and human male infertility. Andrologia 2018.

20. Gu AH, Liang J, Lu NX, Wu B, Xia YK, Lu CC, Song L, Wang SL, Wang XR. Association of XRCC1 gene polymorphisms with idiopathic azoospermia in a Chinese population. Asian J Androl 2007; 781-786.

21. Ji G, Gu A, Zhu P, Xia Y, Zhou Y, Hu F, Song L, Wang S, Wang X. Joint effects of XRCC1 polymorphisms and polycyclic aromatic hydrocarbons exposure on sperm DNA damage and male infertility. Toxicol Sci 2010; 116: 92-98.
22. Matzuk MM, Lamb DJ. Genetic dissection of mammalian fertility pathways. Nat Med 2002; 8: S33.

23. Zhou-Cun A, Yang Y, Zhang SZ, Li N, Zhang W. Single nucleotide polymorphism $\mathrm{C} 677 \mathrm{~T}$ in the methylenetetrahydrofolate reductase gene might be a genetic risk factor for infertility for Chinese men with azoospermia or severe oligozoospermia. Asian J Androl 2007; 9: 57-62.

24. Singh R, Singh L, Thangaraj K. Phenotypic heterogeneity of mutations in androgen receptor gene. Asian J Androl 2007; 9: 147-179.

25. Maduro M, Casella R, Kim E, Levy N, Niederberger C, Lipshultz L, Lamb D. Microsatellite instability and defects in mismatch repair proteins: a new aetiology for Sertoli cell-only syndrome. MHR: Basic Sci Reprod Med 2003; 9: 61-68.

26. Zhoucun A, Zhang S, Yang Y, Ma Y, Zhang W, Lin L. The common variant $\mathrm{N} 372 \mathrm{H}$ in BRCA2 gene may be associated with idiopathic male infertility with azoospermia or severe oligozoospermia. Eur J Obstet Gynecol Reprod Biol 2006; 124: 61-64.

Received February 15, 2019. Accepted March 9, 2019. 